\title{
Adherence to Low-Carbohydrate Diet and Hemoglobin A1c Levels among Adults with Diabetes: A Population-Based Cross-sectional Study
}

\section{Elta Charles and Eunkyung Lee*}

Department of Health Sciences, University of Central Florida, United States of America

*Corresponding Author: Eunkyung Lee, Department of Health Sciences, University of Central Florida, United States of America.
Received: June 30, 2021

Published: July 21, 2021

(C) All rights are reserved by Elta Charles and Eunkyung Lee.

\section{Abstract}

Evidence of a low-carbohydrate diet (LCD) on diabetes prognosis is not consistent. This study examined the association between adherence to LCD and hemoglobin A1c (HbA1c) levels in adults with diabetes. A total of 3,628 adults with self-reported diabetes were identified from the National Health and Nutrition Examination Survey 2005-2016. Adherence to LCD was measured using LCD scores (range 0-30) based on the proportions of energy intake from three macronutrients, with higher scores indicating lower intakes of carbohydrates. Average dietary intakes and HbA1c levels were estimated and compared across the quintiles of LCD scores. Multivariable survey logistic regression models were used to examine the associations between quintile of LCD score and elevated HbA1c levels ( $\geq 6.5 \%$ ). The average diet of adults with DM consisted of $47.2 \%$ carbohydrates, $36.0 \%$ fat, and $16.8 \%$ protein, with a mean LCD score of 15.6. The mean HbA1c level was 7.15\%, and $64 \%$ of participants presented with elevated HbA1c levels of $\geq 6.5 \%$. There was no significant difference in odds of presenting with elevated HbA1c levels when those in the highest and lowest quintiles of LCD were compared after adjustment for potential confounders (OR=0.94; 95\%CI=0.57-1.53). However, individuals in the highest quintile of LCD score showed lower intakes of total fruits, added sugars, and refined grains, but higher intakes of total energy, saturated fats, cholesterol, alcohol, and sodium compared to those in the lowest quintile. Future studies should focus more on diet quality, in addition to the composition of the diet.

Keywords: NHANES: Diabetes Mellitus; Low-carbohydrate Diet; 24-hour Recall; Prognostic Factor; Glycosylated Hemoglobin

\section{Abbreviations}

BMI: Body Mass Index; CI: Confidence Interval; DM: Diabetes Mellitus; HbA1C: Glycosylated Hemoglobin; LCD: Low-carbohydrate Diet; NCHS: National Center for Health Statistics; NHANES: National Health and Nutritional Examination Survey; OR: Odds Ratio; RCT: Randomized Controlled Trial

\section{Introduction}

Among numerous chronic diseases that affect Americans, diabetes mellitus (DM) continues to be of concern. As of 2018, it has been estimated that 34.1 million American adults have DM [1], and the majority (90-95\%) of these cases are type 2 DM [1]. As a result of the increase in cases over the past few decades, preventing complications of DM has become as important as preventing the incidence of DM. When improperly controlled, DM is known to cause microvascular and macrovascular complications due to the severe damage to blood vessels [2].

Complications of DM can be prevented through proper management of blood glucose levels by changing lifestyle and dietary pat- 
terns and by closely monitoring the prognostic factors [3]. Among many prognostic factors, blood glycosylated hemoglobin (HbA1c) level is most widely used in clinics since it is easy to measure and reflects the patient's glycemic controls over the past two to three months [4]. The levels of HbA1c of individuals diagnosed with DM can be heavily dependent on the nature of their regular diet [5]. Carbohydrates are the primary focus for glycemic control because of the nature of their relationship to the glycemic load (i.e., a measure of how much the carbohydrate content of meals raises blood glucose) and the subsequent impact on insulin response [6,7].

Although a low-carbohydrate diet (LCD) is among the many meal plans followed by those with DM [8], evidence of LCD for health outcomes among individuals with DM is scarce and inconsistent. Many studies have been limited to those who are healthy DM but at high risk for type 2 DM [9-11], randomized clinical trials [12-16] which had limited statistical power due to a small sample, or clinical trials with extremely low carbohydrate contents, limiting applications of study findings to community-dwelling individuals with DM. For example, a large cross-sectional study from the United Kingdom was conducted among general population those without DM using a National Diet and Nutrition Survey, and the study reported that every $5 \%$ decrease in energy from carbohydrate was associated with higher, not lower, HbA1c levels [9]. A meta-analysis of four cohort studies reported no evidence of the association between the LCD and the risk for type 2 DM (pooled RR 1.17; 95\% CI 0.90-1.51) with high heterogeneity among studies [11]. Further subgroup analysis revealed that studies with a high quality reported a null association. In contrast, ones with a low quality showed a significant, positive association [11], which warrants more research in this area particularly among individuals with DM in a large community setting.

Thus, this study aims to examine dietary intakes of individuals with DM and the association between the LCD and HbA1c levels using data from the National Health and Nutrition Examination Survey (NHANES), the largest database of a continuous, crosssectional survey for nutrition and health among Americans. Understanding the role of macronutrient composition of diet in glycemic control will help individuals with DM concerned about their diet and prognosis of the disease.

\section{Materials and Methods}

\section{Study design and population}

The NHANES is a cross-sectional survey conducted by the US Centers for Disease Control and Prevention (CDC)'s National Center for Health Statistics (NCHS) to collect data on diet, medical conditions, lifestyle, and health indicators of the US populations via interviews, questionnaires, and examinations [17]. The NHANES started in the 1960s and became a continuous program in 1999. Using a complex multiple-stage cluster probability sampling design, the NHANES selects about 5,000 individuals of all ages and races/ethnicities each year to create a representative sample of civilian, non-institutionalized US residents [17]. Dietary interviews and physical examinations were conducted with medical/ health professionals at the mobile examination center, and some interviews were also conducted in the participant's home using the computer systems [17]. Biological samples are also collected. The National Center for Health Statistics Research Ethics Review Board approved the NHANES. All participants provided informed consent to use their information for future research, including secondary analysis, at the time of data collection [17]. Data is de-identified and made available publicly for research purposes by the NCHS. The current study has been reviewed and approved by the Institutional Review Board of the University of Central Florida (IRB \# SBE-18-14542).

From the NHANES 2005-2016, adults ( $\geq 20$ years old) with DM were identified. DM status was self-reported by the question, "have you ever been told by a doctor or health professional that you have diabetes?" [18]. From the 4,114 eligible participants, individuals were excluded if they were either pregnant or lactating $(n=16)$, had missing data on reliable dietary records $(\mathrm{n}=304)$, or had missing laboratory HbA1c measures $(n=204)$. Since some individuals met more than one exclusion criterion, a total of 486 individuals was excluded, and 3,628 were left for the analysis. All analyses were weighted using the examination sample weights.

Dietary intake and adherence to low-carbohydrate diet (LCD)

The NHANES assessed participant's dietary intakes using 24hour recalls, which were administered by a trained dietary interviewer at the mobile examination centers on one day and a followup interview 7-10 days later via telephone [17]. However, for this study, only the first 24-hour recall was used. Dietary recalls were 
conducted using the US Department of Agriculture (USDA)'s Automated Multiple-Pass Method that probed for any forgotten foods, time, and details of food for complete recalls [19]. The foods and beverages reported in NHANES were converted to the 37 USDA food groups or dietary constituents (e.g., total fruits, whole grains, added sugars) using the USDA Food Patterns Equivalents Database [20].

The LCD score was calculated using the methods provided by Halton., et al. [21] from the daily total calorie/energy intakes and macronutrient compositions. Briefly, the proportion of daily calories from carbohydrates was categorized into 11 groups, and participants in the highest carbohydrate intake group received 0 points, and those in the lowest carbohydrate intake group received 10 points. The same procedures were repeated separately for protein and fat, but the points were reversed; participants in the highest fat/protein intake group received 10 points, and those in the lowest fat/protein intake group received 0 points. Then the points from the three macronutrients were summed together to determine the LCD score, which ranged from 0 to 30 . The score is meant to represent an individual's adherence to an LCD; thus, the highest score indicates that the participant followed the LCD diet more closely and consumed the least proportions of carbohydrates. In this study, LCD scores were further categorized into quintiles, with the $1^{\text {st }}$ quintile representing the lowest LCD scores $(0-8.18)$ and the $5^{\text {th }}$ quintile representing the highest LCD scores $(21.74-30.0)$. The reference group was defined as individuals in the $1^{\text {st }}$ quintile of LCD scores representing those who consumed the highest carbohydrate intake and lowest fat and protein intakes.

\section{Blood HbA1c levels}

The primary outcome was blood HbA1c levels. Blood was collected by venipuncture at NHANES mobile examination centers. Samples were processed, stored at $-20^{\circ} \mathrm{C}$, and shipped to analytic laboratories. The NHANES measured the blood HbA1c levels using the protocol [22] established for the NHANES specimens with the Tosoh Automated Glycohemoglobin Analyzer HLC-723G8 by the Nutritional Biomarkers Branch, the Division of Laboratory Sciences, National Center for Environmental Health, CDC. The HbA1c levels $\geq 6.5 \%$ were categorized as "Elevated," and levels $<6.5 \%$ were classified as "Normal" based on a standardized cut-off point set by the American Diabetes Association [23].

\section{Covariates}

Weight and height were measured by trained health technicians at mobile examination centers. Body mass index (BMI) was calculated as weight in $\mathrm{kg}$ divided by squared height in meter. Sex, age, race/ethnicity, education, marital status, smoking status, household income, and physical activity were self-reported and assessed via interview. The time spent reported on various physical activities, including different intensities of work-related and leisure-time physical activities and transportation (in minutes), was quantified using conversion factors according to varying intensity of activities provided by the NHANES and compared with physical activity recommendation of $150 \mathrm{~min} /$ week [24] to define physical activity adherence. Time since diagnosis was calculated in years using two variables: the age when first told having DM and the age at the survey.

\section{Statistical analysis}

Because multiple years of continuous NHANES data were combined, appropriate subsample weights, clusters, and strata were taken into account following the Analytic and Reporting Guidelines [25]. Descriptive statistics were summarized as frequencies (weighted \%) or weighted mean ( \pm standard error) as appropriate. Demographic characteristics were examined overall and by quintiles of LCD score. The dietary intakes were examined and compared across the quintiles of LCD scores using least-square mean comparisons within the survey regression procedure (SAS proc survey reg). As HbA1c levels are skewed, the geometric means and their standard errors were directly obtained from the SUDAAN proc descript procedure.

Survey logistic regression analyses were used to estimate odds of presenting elevated HbA1c by quintiles of LCD scores. The demographics-adjusted model included sex (male and female), age (year, continuous), and race/ethnicity (non-Hispanic white, nonHispanic black, Mexican American, and other). The multivariable model was further adjusted for total daily energy (kcal, continuous), food group (e.g., total fruits, whole grains, added sugars, continuous), BMI $\left(<25,25-<30\right.$, and $\left.\geq 30 \mathrm{~kg} / \mathrm{m}^{2}\right)$, income $(<\$ 20,000$, $\$ 20,000-<\$ 75,000, \geq \$ 75,000$ ), marital status (married/partner, widowed, divorced/separated), smoking status (never, former, and current), time since diagnosis (year, continuous), and adherence to physical activity recommendation (below, met, and exceed). Unad- 
justed and adjusted odds ratios (ORs) and corresponding 95\% confidence intervals (CIs) were reported, and statistical significance was set at $P<0.05$. The data were analyzed using SAS (version 9.4, SAS Institute, Inc., Cary, NC) [26] and SUDAAN (version 13, RTI International, Research Triangle Park, NC) [27].

\section{Results and Discussion}

Participants

The final sample included 3,628 adults with DM from the NHANES 2005-2016. Participant demographic characteristics are shown in table 1 . About half of the individuals with DM were male. The majority of adults with DM were age 40 years and older (92.3\%), non-Hispanic whites (61.9\%), overweight or obese (88.4\%), graduates of high school or above (74.7\%), married/living with a partner $(63.3 \%)$, never or former smokers (84.3\%), and earning $\geq \$ 20,000 /$ year (77.3\%). Half of them did not meet the physical activity recommendations (53.8\%). Those in the highest quintile were more likely to be male, younger, non-Hispanic whites, married, and of higher income than those in the lowest quintile.

\begin{tabular}{|c|c|c|c|c|c|c|c|c|c|}
\hline \multirow[b]{2}{*}{ Characteristics } & \multicolumn{9}{|c|}{ Quintiles of Low-Carbohydrate Diet (LCD) Score } \\
\hline & \multirow[b]{2}{*}{$\mathbf{n}$} & \multirow{2}{*}{\begin{tabular}{|c} 
Overall $\mathrm{N}=$ \\
3,628
\end{tabular}} & \multirow[b]{2}{*}{$\%$} & $\begin{array}{c}\text { Lowest } \\
\text { Q1 } \\
\text { (n= 799) }\end{array}$ & $\begin{array}{c}\text { Second } \\
\text { Q2 (n = } \\
766)\end{array}$ & $\begin{array}{l}\text { Middle Q3 } \\
(n=628)\end{array}$ & $\begin{array}{c}\text { Fourth Q4 } \\
(n=644)\end{array}$ & $\begin{array}{c}\text { Highest } \\
\text { Q5 (n = } \\
791)\end{array}$ & \multirow[t]{2}{*}{ P-Value ${ }^{\text {a }}$} \\
\hline & & & & \multicolumn{5}{|c|}{ n (weighted \%) } & \\
\hline \multicolumn{10}{|l|}{ Sex } \\
\hline Male & 1,853 & $9,345,331$ & 50.6 & $322(40.0)$ & $\begin{array}{c}353 \\
(45.8)\end{array}$ & $321(49.0)$ & $374(57.1)$ & $483(59.1)$ & $<.0001$ \\
\hline Female & 1,775 & $9,135,916$ & 49.4 & $477(60.0)$ & $\begin{array}{c}413 \\
(54.2)\end{array}$ & 307 (51.0) & $270(42.9)$ & $308(40.9)$ & \\
\hline \multicolumn{10}{|l|}{ Age (year) } \\
\hline $20-39$ & 223 & $1,427,359$ & 7.7 & $44(7.3)$ & $39(7.0)$ & $38(8.3)$ & $42(6.8)$ & $60(9.0)$ & 0.025 \\
\hline $40-59$ & 1,156 & $7,086,046$ & 38.4 & $248(38.8)$ & $\begin{array}{c}224 \\
(33.3)\end{array}$ & $191(37.4)$ & $211(36.2)$ & $282(44.7)$ & \\
\hline$\geq 60$ & 2,249 & $9,967,842$ & 53.9 & $507(53.9)$ & $\begin{array}{c}503 \\
(59.7) \\
\end{array}$ & $399(54.3)$ & $391(57.0)$ & 449 (46.3) & \\
\hline \multicolumn{10}{|l|}{$\begin{array}{l}\text { Body mass index } \\
\left(\mathrm{kg} / \mathrm{m}^{2}\right)\end{array}$} \\
\hline$<25.0$ & 781 & $2,117,758$ & 11.6 & 110 (11.9) & $\begin{array}{c}103 \\
(13.4)\end{array}$ & $79(11.0)$ & $77(10.6)$ & $95(11.4)$ & 0.523 \\
\hline $25.0-<30.0$ & 1,009 & $4,738,553$ & 26.1 & $211(25.2)$ & $\begin{array}{c}220 \\
(27.6)\end{array}$ & 177 (28.3) & $179(27.5)$ & $222(22.7)$ & \\
\hline$\geq 30.0$ & 2,074 & $11,310,635$ & 62.3 & 452 (62.9) & $\begin{array}{c}424 \\
(59.0)\end{array}$ & 355 (60.7) & $379(61.9)$ & $464(65.9)$ & \\
\hline \multicolumn{10}{|l|}{ Race/ethnicity } \\
\hline Non-Hispanic White & 1,300 & $11,443,930$ & 61.9 & $214(52.1)$ & $\begin{array}{c}280 \\
(62.4)\end{array}$ & $230(63.7)$ & $285(70.2)$ & $291(61.4)$ & $<.0001$ \\
\hline Non-Hispanic Black & 978 & $2,871,838$ & 15.5 & $232(19.4)$ & $\begin{array}{c}182 \\
(14.6)\end{array}$ & $179(15.3)$ & $131(10.9)$ & $254(17.1)$ & \\
\hline Mexican American & 685 & $1,735,354$ & 9.4 & $164(11.1)$ & $153(9.6)$ & $108(8.9)$ & $125(8.4)$ & $135(8.9)$ & \\
\hline
\end{tabular}




\begin{tabular}{|c|c|c|c|c|c|c|c|c|c|}
\hline Other & 665 & $2,430,125$ & 13.2 & $189(14.4)$ & $\begin{array}{c}151 \\
(13.4) \\
\end{array}$ & $111(12.0)$ & $103(10.5)$ & $111(12.6)$ & 48 \\
\hline \multicolumn{10}{|l|}{ Education } \\
\hline$<12^{\text {th }}$ grade & 1,323 & $4,669,409$ & 25.3 & $339(30.3)$ & $\begin{array}{c}285 \\
(26.1) \\
\end{array}$ & $211(25.7)$ & $224(22.3)$ & $264(22.5)$ & 0.213 \\
\hline High School Graduate & 832 & $4,561,635$ & 24.7 & $175(25.2)$ & $\begin{array}{c}180 \\
(23.8)\end{array}$ & $161(25.9)$ & $145(24.6)$ & $171(24.3)$ & \\
\hline Some college & 934 & $5,774,103$ & 31.2 & $168(26.1)$ & $\begin{array}{c}203 \\
(33.8) \\
\end{array}$ & $172(32.0)$ & $165(31.8)$ & $226(32.3)$ & \\
\hline$\geq$ College graduate & 534 & $3,469,196$ & 18.8 & $115(18.4)$ & $97(16.3)$ & $82(16.4)$ & $110(21.3)$ & $130(20.9)$ & \\
\hline \multicolumn{10}{|l|}{ Marital status } \\
\hline Married/Partner & 2,156 & $11,680,024$ & 63.3 & $429(59.0)$ & $\begin{array}{c}466 \\
(63.0) \\
\end{array}$ & $353(57.2)$ & $404(67.2)$ & $504(68.3)$ & 0.012 \\
\hline Widowed & 533 & $2,199,950$ & 11.9 & $138(13.1)$ & $\begin{array}{c}122 \\
(14.4)\end{array}$ & $102(12.5)$ & $91(12.1)$ & $80(8.2)$ & \\
\hline Divorced/Separated & 619 & $2,907,931$ & 15.8 & $157(18.6)$ & $\begin{array}{c}116 \\
(13.7)\end{array}$ & 113 (18.2) & $95(13.3)$ & $138(15.4)$ & \\
\hline Single & 317 & $1,667,871$ & 9.0 & $73(9.3)$ & $62(8.9)$ & $60(12.1)$ & $54(7.4)$ & $68(8.1)$ & \\
\hline \multicolumn{10}{|l|}{ Smoking status } \\
\hline Never & 1,811 & $9,057,250$ & 49.0 & $438(51.7)$ & $\begin{array}{c}401 \\
(52.2) \\
\end{array}$ & $314(50.2)$ & $287(43.8)$ & $371(47.5)$ & 0.160 \\
\hline Former & 1,248 & $6,526,150$ & 35.3 & $232(30.4)$ & $\begin{array}{c}262 \\
(34.3) \\
\end{array}$ & $220(34.8)$ & $248(40.4)$ & $286(36,4)$ & \\
\hline Current & 568 & $2,896,679$ & 15.7 & 129 (17.9) & $\begin{array}{c}103 \\
(13.5)\end{array}$ & $94(15.0)$ & $109(15.8)$ & $133(16.1)$ & \\
\hline \multicolumn{10}{|l|}{ Income } \\
\hline$<\$ 20,000$ & 1,069 & $4,064,120$ & 22.7 & $280(28.2)$ & $\begin{array}{c}245 \\
(27.5) \\
\end{array}$ & $178(22.5)$ & $174(21.1)$ & $192(15.9)$ & $<.0001$ \\
\hline$\$ 20,000-\$ 75,000$ & 1,859 & $9,812,944$ & 54.9 & $388(54.0)$ & $\begin{array}{c}387 \\
(54.5)\end{array}$ & $319(55.0)$ & $329(51.3)$ & $436(58.8)$ & \\
\hline$\geq \$ 75,000$ & 544 & $4,002,251$ & 22.4 & $91(17.8)$ & $97(18.0)$ & $100(22.5)$ & $123(27.6)$ & $133(25.3)$ & \\
\hline \multicolumn{10}{|l|}{$\begin{array}{l}\text { Physical Activity } \\
\text { Adherence }^{\mathrm{b}}\end{array}$} \\
\hline Below & 2,103 & $9,945,772$ & 53.8 & $494(56.5)$ & $\begin{array}{c}454 \\
(54.3) \\
\end{array}$ & 355 (53.6) & $363(54.0)$ & $437(51.2)$ & 0.730 \\
\hline Met & 351 & $1,969,002$ & 10.7 & $76(10.7)$ & 75 (12.1) & $58(8.6)$ & $64(10.1)$ & 78 (11.4) & \\
\hline Exceed & 1174 & $6,566,473$ & 35.5 & $229(32.8)$ & $\begin{array}{c}237 \\
(33.6) \\
\end{array}$ & 215 (37.8) & 217 (35.9) & $276(37.4)$ & \\
\hline $\begin{array}{l}\text { a P-values are from on } \\
{ }^{\mathrm{b}} \text { Based on physical act }\end{array}$ & i-square & tests. Signifi & ant fin & $\begin{array}{l}\text { dings are bol } \\
\text { n/week }\end{array}$ & & & & & \\
\hline
\end{tabular}

Table 1: Characteristics of adults aged 20 and older with diabetes mellitus by quintiles of low-carbohydrate diet score, National Health and Nutrition Examination Survey, 2005-2016. 
Dietary intakes overall and by LCD score quintiles

Table 2 presents dietary intakes among American adults with DM overall and by the quintiles of LCD score. The average diet consisted of $47.2 \%$ carbohydrates, $36.0 \%$ fat, and $16.8 \%$ protein, and the estimated mean LCD score of adults with DM was $15.6( \pm$ 0.17 ). As shown in figure 3 , saturated fat, refined grains, added sugars, and sodium intake exceeds recommended dietary intakes for American adults, while dairy, fruit and vegetable intakes were below the recommended. Daily energy intake, diet composition, and nutrient intakes were significantly different by the quintiles of LCD score. Those in the highest quintile of LCD score consumed signifi- cantly higher energy per day, greater proportions from protein, fat, and saturated fat than those in the lowest quintile. Those in the highest quintile also consumed significantly less added sugars but a greater amount of cholesterol and alcohol than those in the lowest quintile of LCD score. When comparing the dietary constituents per $1000 \mathrm{kcal}$, those in the highest quintile consumed significantly more seafood and plant proteins and sodium, but less total fruits. There were no significant differences in intakes of total vegetables, dark green vegetables and beans, and dairy products between the highest and lowest quintiles of LCD scores.

\begin{tabular}{|c|c|c|c|c|c|c|c|}
\hline \multirow[b]{2}{*}{ Variables } & \multicolumn{7}{|c|}{ Quintiles of Low-Carbohydrate Diet (LCD) Score } \\
\hline & $\begin{array}{c}\text { Overall } \\
N=3,628\end{array}$ & Lowest Q1 & Second Q2 & Middle Q3 & Fourth Q4 & Highest Q5 & $P_{Q 5 \text { vs. Q1 }}{ }^{\mathrm{a}}$ \\
\hline & \multicolumn{7}{|c|}{ Mean $\left(\mathrm{SE}^{\mathrm{b}}\right)$} \\
\hline LCD score & $15.6(0.17)$ & $4.8(0.13)$ & $11.2(0.06)$ & $15.5(0.05)$ & $19.5(0.07)$ & $24.8(0.11)$ & $<.001$ \\
\hline Energy (kcal) & $1913.8(20.61)$ & $\begin{array}{l}1726.1 \\
(43.69)\end{array}$ & $1820.9(42.69)$ & $1915.1(57.81)$ & $2035.8(50.54)$ & $2044.7(47.05)$ & $<.001$ \\
\hline Carbohydrate (\% energy) & $47.2(0.26)$ & $62.6(0.38)$ & $53.0(0.18)$ & $47.6(0.11)$ & $41.5(0.26)$ & $33.9(0.28)$ & $<.001$ \\
\hline Protein (\% energy) & $16.8(0.13)$ & $12.7(0.15)$ & $15.6(0.20)$ & $16.8(0.22)$ & $17.2(0.26)$ & $20.8(0.30)$ & $<.001$ \\
\hline Fat (\% energy) & $36.0(0.24)$ & $24.7(0.36)$ & $31.3(0.35)$ & $35.6(0.30)$ & $41.3(0.40)$ & $45.3(0.39)$ & $<.001$ \\
\hline Saturated Fat (\% energy) & $11.5(0.09)$ & $9.5(0.16)$ & $10.4(0.12)$ & $11.8(0.19)$ & $12.4(0.17)$ & $13.2(0.16)$ & $<.001$ \\
\hline Added sugars (\% energy) & $9.7(0.17)$ & $14.8(0.49)$ & $10.6(0.34)$ & $9.7(0.30)$ & $7.8(0.23)$ & $6.5(0.21)$ & $<.001$ \\
\hline Fatty acid ratioc & $1.9(0.02)$ & $1.9(0.03)$ & $2.0(0.03)$ & $1.9(0.04)$ & $1.9(0.03)$ & $2.0(0.03)$ & 1.000 \\
\hline Cholesterol (mg) & $296.1(6.41)$ & $\begin{array}{r}160.3 \\
(8.11)\end{array}$ & $217.9(8.58)$ & $275.3(10.51)$ & $332.5(11.23)$ & $459.7(13.25)$ & $<.001$ \\
\hline Total sugars $(\mathrm{gm})$ & $88.6(1.49)$ & $\begin{array}{l}131.5 \\
(5.54)\end{array}$ & $100.2(3.02)$ & $86.9(2.65)$ & $73.2(2.28)$ & $57.5(1.92)$ & $<.001$ \\
\hline Alcohol (gm) & $5.3(0.49)$ & $1.2(0.26)$ & $2.2(0.49)$ & $5.1(0.93)$ & $10.4(1.94)$ & $7.4(1.09)$ & $<.001$ \\
\hline Total fruits $\left(\mathrm{c}-\mathrm{eq}^{\mathrm{d}}\right)$ & $0.54(0.01)$ & $0.81(0.03)$ & $0.67(0.04)$ & $0.51(0.03)$ & $0.43(0.02)$ & $0.34(0.02)$ & $<.001$ \\
\hline Total vegetables (c-eq) & $0.95(0.02)$ & $1.00(0.04)$ & $1.01(0.03)$ & $0.92(0.03)$ & $0.91(0.03)$ & $0.92(0.03)$ & 0.507 \\
\hline $\begin{array}{l}\text { Dark green vegetables \& } \\
\text { beans (c-eq) }\end{array}$ & $0.14(0.01)$ & $0.17(0.02)$ & $0.14(0.01)$ & $0.14(0.01)$ & $0.12(0.01)$ & $\begin{array}{c}0.14 \\
(0.01)\end{array}$ & 0.705 \\
\hline Dairy (c-eq) & $0.75(0.01)$ & $0.69(0.02)$ & $0.77(0.02)$ & $0.83(0.03)$ & $0.76(0.03)$ & $0.70(0.03)$ & 0.998 \\
\hline $\begin{array}{l}\text { Seafood and plant proteins } \\
\left(\mathrm{oz}^{-} \mathrm{eq}^{\mathrm{e}}\right)\end{array}$ & $0.97(0.03)$ & $0.80(0.04)$ & $0.95(0.06)$ & $0.98(0.06)$ & $0.96(0.06)$ & $1.11(0.06)$ & $<.001$ \\
\hline Refined grains (oz-eq) & $2.8(0.03)$ & $2.9(0.07)$ & $2.9(0.05)$ & $2.9(0.07)$ & $2.8(0.05)$ & $2.5(0.05)$ & $<.001$ \\
\hline Sodium (gm/1000 kcal) & $1.8(0.01)$ & $1.7(0.02)$ & $1.8(0.02)$ & $1.8(0.02)$ & $1.9(0.02)$ & $2.0(0.03)$ & $<.001$ \\
\hline $\begin{array}{l}\text { P values are from least squ } \\
\text { b } \mathrm{SE}=\text { standard error } \\
\text { cratio of unsaturated fats to } \\
{ }^{\mathrm{d}} \text { c-eq=cup equivalent } / 1000 \\
{ }^{\mathrm{o}} \text { oz-eq=ounce equivalent } / 10\end{array}$ & $\begin{array}{l}\text { re means compar } \\
\text { aturated fats } \\
\text { cal } \\
00 \mathrm{kcal}\end{array}$ & rison after ac & justing for multi & ple comparison & s. Significant fin & dings are bold. & \\
\hline
\end{tabular}

Table 2: Dietary intakes of adults aged 20 and older with diabetes mellitus overall and by quintiles of low-carbohydrate diet score, National Health and Nutrition Examination Survey, 2005-2016. 
Mean HbA1c and prevalence of elevated levels by the quintiles of LCD score

As shown in figure 1, the estimated geometric mean $\mathrm{HbA1c}$ level in adults with DM was $7.15( \pm 0.036) \%$, with a significantly higher mean level in males than females $(7.22 \%$ vs. $7.07 \%, p=0.026)$. There were no significant differences by the quintiles of LCD score overall and for males and females separately. Figure 2 presents the proportions of adults with elevated HbA1c levels. About $64 \%$ of adults with DM were estimated to have $\mathrm{HbA1c} \geq 6.5 \%$, with a significantly higher proportion in adult males than in females $(67.0 \%$ vs. $61.0 \%, \mathrm{p}=0.009$ ). There were no significant differences in the prevalence of elevated HbA1C across LCD score quintiles in both sexes evaluated together and in males and females separately.

\section{Between LCD score and elevated HbA1c levels}

As shown in table 3 , the odds of presenting with elevated HbA1c levels were not significantly different between those in the highest quintile of LCD and those in the lowest quintile $\left(\mathrm{OR}_{\mathrm{q} 5 \mathrm{vsq} 1}=1.25\right.$, $\left.95 \% \mathrm{CI}=0.91-1.72, \mathrm{P}_{\text {trend }}=0.595\right)$. After adjustment for age, sex, and race/ethnicity, the odds ratios (OR) were slightly decreased across the $\mathrm{LCD}$ quintiles but still not significant $\mathrm{OOR}_{\mathrm{q} 5 \mathrm{vsq} 1}=1.22,95 \% \mathrm{CI}=$ $0.88-1.68, P_{\text {trend }}=0.719$ ). Further, the multivariate analysis includ-

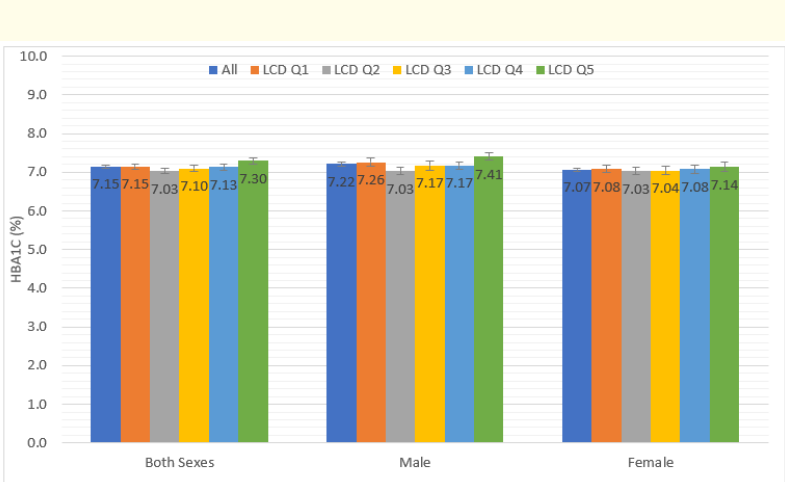

Figure 1: Geometric mean blood HbA1c levels by the quintiles of low-carbohydrate diet (LCD) score among adults with diabetes, National Health and Nutrition Examination Survey, 2005-2016.

Q1: $1^{\text {st }}$ quintile of LCD score, Q2: $2^{\text {nd }}$ quintile, Q3: $3^{\text {rd }}$ quintile, Q4: $4^{\text {th }}$ quintile, and Q5: $5^{\text {th }}$ quintile.

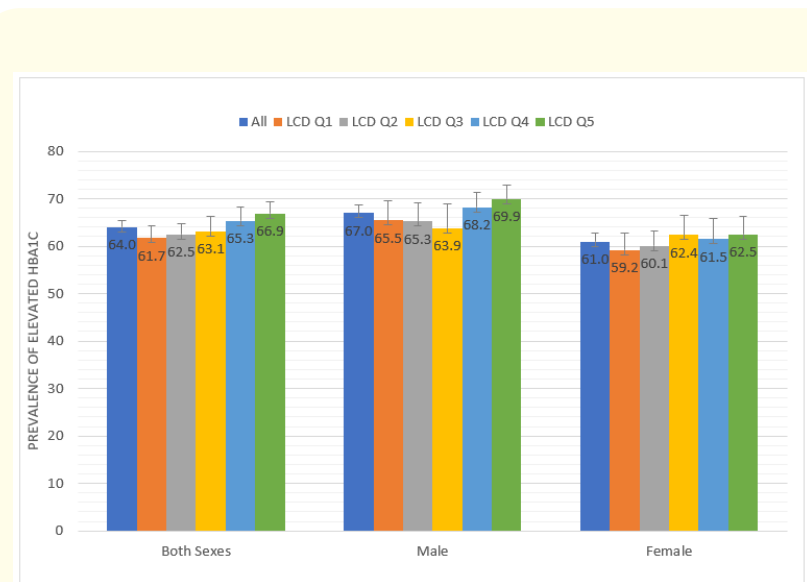

Figure 2: Prevalence of elevated HbA1c levels by the quintiles of low-carbohydrate diet (LCD) score in adults with diabetes mellitus (DM), National Health and Nutrition Examination Survey, 2005-2016.

Q1: $1^{\text {st }}$ quintile of LCD score, Q2: $2^{\text {nd }}$ quintile, Q3: $3^{\text {rd }}$ quintile, Q4: $4^{\text {th }}$ quintile, and Q5: $5^{\text {th }}$ quintile.

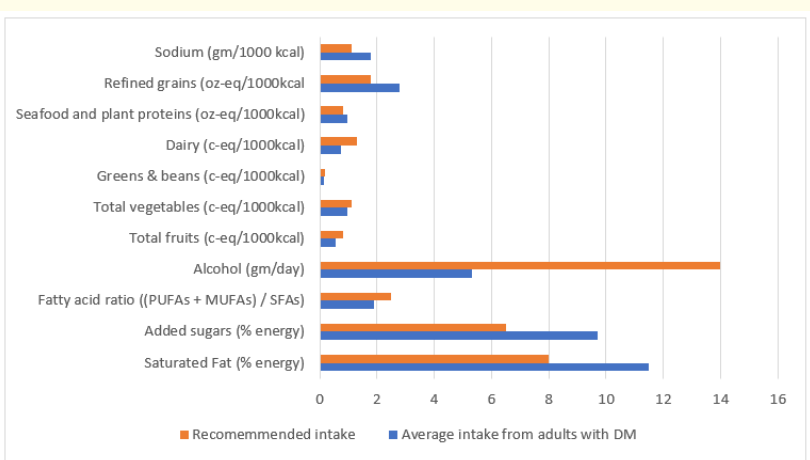

Figure 3: Average dietary intakes of adults with diabetes.

ing all potential confounders showed no associations between the quintiles of LCD scores and elevated HbA1c levels in adults with $\mathrm{DM}\left(\mathrm{OR}_{\mathrm{q} 5 \mathrm{vsq} 1}=0.94,95 \% \mathrm{CI}=0.57-1.53, \mathrm{P}_{\text {trend }}=0.982\right)$. The stratified analysis by gender also showed no associations between the quintiles of LCD scores and elevated HbA1c levels in males and females separately. 


\begin{tabular}{|c|c|c|c|c|c|c|}
\hline & \multicolumn{6}{|c|}{ Quintiles of Low-Carbohydrate Diet Score } \\
\hline & Q1 & Q2 & Q3 & Q4 & Q5 & $P$ for trend \\
\hline \multicolumn{7}{|l|}{ Both sexes } \\
\hline Unadjusted OR (95\% CI) & 1.0 & $1.03(0.77-1.39)$ & $1.06(0.75-1.51)$ & $1.17(0.86-1.58)$ & $1.25(0.91-1.72)$ & 0.595 \\
\hline $\begin{array}{l}\text { Demographics-adjusted OR } \\
(95 \% \mathrm{CI})^{\mathrm{b}}\end{array}$ & 1.0 & $1.04(0.77-1.41)$ & $1.07(0.74-1.53)$ & $1.16(0.86-1.57)$ & $1.22(0.88-1.68)$ & 0.719 \\
\hline $\begin{array}{l}\text { Multivariable-adjusted OR } \\
(95 \% \mathrm{CI})^{\mathrm{c}}\end{array}$ & 1.0 & $0.93(0.67-1.30)$ & $0.90(0.58-1.40)$ & $0.91(0.62-1.34)$ & $0.94(0.57-1.53)$ & 0.982 \\
\hline \multicolumn{7}{|l|}{ Males } \\
\hline Unadjusted OR (95\% CI) & 1.0 & $0.99(0.60-1.63)$ & $0.93(0.53-1.63)$ & $1.13(0.74-1.72)$ & $1.22(0.78-1.94)$ & 0.818 \\
\hline $\begin{array}{l}\text { Demographics-adjusted OR } \\
(95 \% \mathrm{CI})^{\mathrm{b}}\end{array}$ & 1.0 & $1.00(0.60-1.64)$ & $0.93(0.53-1.63)$ & $1.15(0.75-1.76)$ & $1.22(0.78-1.93)$ & 0.813 \\
\hline $\begin{array}{l}\text { Multivariable-adjusted OR } \\
(95 \% \mathrm{CI})^{\mathrm{c}}\end{array}$ & 1.0 & $0.75(0.44-1.30)$ & $0.56(0.28-1.14)$ & $0.63(0.34-1.16)$ & $0.59(0.28-1.27)$ & 0.549 \\
\hline \multicolumn{7}{|l|}{ Females } \\
\hline Unadjusted OR (95\% CI) & 1.0 & $1.04(0.71-1.52)$ & $1.14(0.74-1.76)$ & $1.10(0.70-1.73)$ & $1.15(0.73-1.82)$ & 0.964 \\
\hline $\begin{array}{l}\text { Demographics-adjusted OR } \\
(95 \% \mathrm{CI})^{\mathrm{b}}\end{array}$ & 1.0 & $1.07(0.73-1.58)$ & $1.19(0.77-1.86)$ & $1.17(0.74-1.86)$ & $1.18(0.74-1.88)$ & 0.918 \\
\hline $\begin{array}{l}\text { Multivariable-adjusted OR } \\
(95 \% \mathrm{CI})^{\mathrm{c}}\end{array}$ & 1.0 & $1.11(0.72-1.73)$ & $1.29(0.75-2.24)$ & $1.25(0.68-2.31)$ & $1.40(0.69-2.84)$ & 0.887 \\
\hline \multicolumn{3}{|c|}{ Elevated HbA1c level was defined as blood HbA1c levels $\geq 6.5 \%$} & \multicolumn{4}{|c|}{$\begin{array}{l}\text { bedjusted for sex (male and female), age (year, continuous), and race/ethnicity (Non-Hispanic White, Non-Hispanic Black, Mexican } \\
\text { American, and Other) }\end{array}$} \\
\hline
\end{tabular}

Table 3: Odds ratios of elevated $\mathrm{HbA} 1 \mathrm{c}^{\mathrm{a}}$ by quintiles of low-carbohydrate diet scores in adults ages 20 and older with diabetes mellitus in National Health and Nutritional Examination Survey, 2005-2016.

This study is one of the first studies to examine the relationship between LCD and HbA1c levels among adults with DM at the population level. The findings showed that the LCD score was not associated with the odds of presenting with elevated $\mathrm{HbA1c}$ levels among adults with DM. Additionally, the results indicated that adults with DM consumed more sodium, refined grains, added sugars, and saturated fats, and less dairy, fruit and vegetables than the recommended intake, with more deviations in the highest quintiles of LCD scores. These findings have important clinical and public health implications, as more individuals with DM are adapting LCD for their glucose control [8].
The average percentage of carbohydrates in total energy $(47.3 \%)$ from our sample is within the recommended proportions of carbohydrates (45-60\%) for adults with DM [28]. It is estimated that about $40 \%$ of American adults with DM follow an LCD (Table 2 ) if the most liberal definition ( $<45 \%$ of total energy intake from carbohydrate) of an LCD is used. The macronutrient distribution found for each quintile of LCD score seemed reasonable according to the definition of LCD score. However, it is surprising that total energy, saturated fats, cholesterol, alcohol, and sodium intakes were higher in the overall sample, and these deviations were most 
observed in the highest quintiles of LCD. The American Diabetes Association defines an LCD as one in which highly processed carbohydrate foods and grains are limited or avoided, and the focus shifts towards consumption of non-starchy vegetables and protein foods [29]. Controlling blood glucose levels would call for a decrease in carbohydrate consumption, but it should not lead to the elimination of essential foods such as fruits and other nutrientdense vegetables and whole-grain foods [30]. It is possible that the differences in socioeconomic status could influence food choices among adults with DM as those in the highest quintile were more likely to be of higher income, married, and white.

An examination of mean HbA1c levels across the LCD score quintiles indicated no significant differences, which is consistent with current literature reporting a null association between a lowcarbohydrate diet and glycemic controls or risk for DM among individuals without DM [9-11]. It was difficult to find past research studies examining the same association between LCD score and clinical factors such as HbA1c levels among persons with DM. Most studies have investigated the effect of restriction of carbohydrate intake on HbA1c levels in a clinical setting [12-16]. However, these results should be interpreted with caution because most of the studies were small and had a short-term follow-up. For instance, results from a randomized controlled trial (RCT) in China $(n=58)$ showed a more significant decrease in HbA1c levels in the LCD group at a three-month follow-up when compared with the low-fat diet group [16]. Moreover, most of the studies that found benefits of LCD on glycemic control tested diets in which a very low-carbohydrate diet was used. For example, Sainsbury., et al. conducted a systematic review and meta-analysis of 25 RCT studies examining the effect of carbohydrate restriction on glycemic control for adults with DM [31]. They found that HbA1c levels were reduced, mainly when carbohydrates were restricted to less than $26 \%$ of energy, and the effects were measured at 3-month or 6-month follow-ups. They further reported that studies with moderate carbohydrate reduction of $26-45 \%$ did not show any differences in HbA1c decrease at any time, which may explain why there is no association between LCD score and HbA1c in the current study. Therefore, future studies are needed to examine the glycemic outcomes among those who consume carbohydrate $<26 \%$.

Another possible explanation for a null association in the current study can be found in Halton., et al. [10]. The authors reported an $18 \%$ reduced risk of type $2 \mathrm{DM}$ among the highest quantile of LCD group than the lowest group only when LCD score was elaborated with the source of protein and fat (i.e., animal or vegetable). Hu., et al. [32] reported that a poor diet, such as ones that include foods with higher glycemic load and high intakes of trans fats, was associated with a significantly higher risk of DM, even after adjusting for BMI. Another study found that just a 1-standard deviation increase in diet quality indices was associated with a 9-13\% reduced risk of type $2 \mathrm{DM}$ in men [33]. Among the diet quality indices examined, most of them that showed a significant association with a lower risk of type 2 DM followed common dietary patterns, which were characterized with (i) low intake of refined sugar, meats, sodium, and trans-fats, (ii) high intake of plant-based foods and grains, and (iii) moderate alcohol consumption [33]. These studies all implied that both the proportion of carbohydrates and the quality of other dietary components could be essential in controlling blood glucose levels and insulin resistance in individuals with DM.

There are both limitations and strengths of this study. This study used data from a cross-sectional study; therefore, it cannot determine a temporal relationship. However, it can provide a snapshot of the average dietary intakes of adults with DM and the prevalence of health conditions. Next, participant's dietary intakes and DM status were self-reported, which may introduce the possibility of recall bias and misclassification bias [34]. However, a high correlation was found between the comorbidities reported on the questionnaires and those extracted from medical records [35,36], and these biases are more likely to be non-differential and bias towards the null. Lastly, the sources of protein and fat (plant or animal), which can influence the association between LCD score and HbA1c, were not available from NHANES. To account for this limitation, we examined the food group intakes according to the quintiles of LCD score, and the multivariable analysis model included these food group intakes along with total energy and multiple covariates.

Despite these limitations, this study has several strengths. This study adds to our knowledge base by being the initial, community-based population study addressing the association of LCD and HbA1c levels among individuals with DM. Another strength of this study is that data were obtained from six cycles of the NHANES (2005-2016) leading to a large, nationally representative sample. Therefore, this study had sufficient statistical power to detect an association when, in fact, there was one, and the results are more

Citation: Elta Charles and Eunkyung Lee. "Adherence to Low-Carbohydrate Diet and Hemoglobin A1c Levels among Adults with Diabetes: A Population-Based Cross-sectional Study". Acta Scientific Nutritional Health 5.8 (2021): 44-54. 
generalizable than studies from a small clinical setting. Lastly, in addition to the primary prognostic factor (i.e., HbA1c), food groups were also analyzed. The results suggest that individuals with DM need to pay more attention to achieve a healthy and balanced diet while achieving glycemic control.

\section{Conclusion}

In summary, the results of this study showed that high LCD scores (i.e., low in carbohydrate and high in protein and fat intakes) were not associated with odds of presenting with elevated HbA1c levels among adults with DM. The current study, along with other studies, suggests that the proportion of carbohydrates in one's diet may not be the only factor influencing the HbA1c levels. Nutritional education programs need to emphasize the importance of a healthy and balanced diet in glycemic control, including total energy, quality and quantity of three macronutrients, and the role of other micronutrients such as vitamins, minerals, and fiber. Future studies examining the role of diet quality in glycemic controls among individuals with DM will provide practical information to improve the disease outcomes of individuals with DM.

\section{Acknowledgements}

The authors would like to thank the participants in the NHANES surveys and acknowledge the efforts of the Centers for Disease Control and Prevention/National Center for Health Statistics.

\section{Author Contributions}

EC and EL designed the study. EL was responsible for the statistical analysis. EL and EC reviewed the data analysis. EC drafted the manuscript as her undergraduate honors thesis and EL revised the manuscript for publication. All authors critically reviewed the manuscript and approved the final version submitted for publication.

\section{Conflict of Interest}

The authors declare that they have no conflict interest.

\section{Bibliography}

1. Centers for Disease Control and Prevention. "National Diabetes Statistics Report 2020: Estimates of Diabetes and Its Burden in the United States" (2020).
2. Fowler MJ. "Microvascular and macrovascular complications of diabetes". Clinical Diabetes 26.2 (2008): 77-82.

3. Asif M. "The prevention and control the type-2 diabetes by changing lifestyle and dietary pattern". Journal of Education and Health Promotion 3 (2014): 1.

4. Sherwani SI., et al. "Significance of HbA1c Test in Diagnosis and Prognosis of Diabetic Patients". Biomark Insights 11 (2016): 95-104.

5. Sami W., et al. "Type 2 diabetes mellitus: Link between diet, HbA1c and complications". Australasian Medical Journal (Online) 9.9 (2016): 346-356.

6. Oregon State University: Linus Pauling Institute. Glycemic Index and Glycemic Load (2003).

7. Riccardi G., et al. "Role of glycemic index and glycemic load in the healthy state, in prediabetes, and in diabetes". The American Journal of Clinical Nutrition 87.1 (2008): 269S-274S.

8. Dyson P. "Low carbohydrate diets and type 2 diabetes: What is the latest evidence?" Diabetes Therapy 6.4 (2015): 411-424.

9. Churuangsuk C., et al. "Lower carbohydrate and higher fat intakes are associated with higher hemoglobin A1c: findings from the UK National Diet and Nutrition Survey 2008-2016". European Journal of Nutrition 59.6 (2020): 2771-2782.

10. Halton TL., et al. "Low-carbohydrate-diet score and risk of type 2 diabetes in women". The American Journal of Clinical Nutrition 87.2 (2008): 339-346.

11. Namazi N., et al. "Low-Carbohydrate-Diet Score and its Association with the Risk of Diabetes: A Systematic Review and MetaAnalysis of Cohort Studies". Hormone and Metabolic Research 49.8 (2017): 565-571.

12. Tay J., et al. "Comparison of low- and high-carbohydrate diets for type 2 diabetes management: a randomized trial". The American Journal of Clinical Nutrition 102.4 (2015): 780-790.

13. Yamada Y., et al. "A Non-calorie-restricted Low-carbohydrate Diet is Effective as an Alternative Therapy for Patients with Type 2 Diabetes". Internal Medicine 53.1 (2014): 13-19. 
14. Guldbrand H., et al. "In type 2 diabetes, randomisation to advice to follow a low-carbohydrate diet transiently improves glycaemic control compared with advice to follow a low-fat diet producing a similar weight loss". Diabetologia 55.8 (2012): 2118-2127.

15. Daly ME., et al. "Short-term effects of severe dietary carbohydrate-restriction advice in Type 2 diabetes--a randomized controlled trial". Diabetic Medicine 23.1 (2006): 15-20.

16. Wang LL., et al. "The Effect of Low-Carbohydrate Diet on Glycemic Control in Patients with Type 2 Diabetes Mellitus". Nutrients 10.6 (2018).

17. Centers for Disease Control and Prevention. About the National Health and Nutrition Examination Survey. National Health and Nutrition Examination Survey (2018).

18. Centers for Disease Control and Prevention. National Health and Nutrition Examination Survey: 2015-2016 Data Documentation, Codebook, and Frequencies (2017).

19. Steinfeldt L., et al. "Food reporting patterns in the USDA Automated Multiple-Pass Method". 36th National Nutrient Databank Conference 2 (2013): 145-156.

20. US Department of Agriculture. "Food Patterns Equivalents Database" (2019).

21. Halton TL., et al. "Low-carbohydrate-diet score and the risk of coronary heart disease in women". New England Journal of Medicine 355.19 (2006): 1991-2002.

22. Little R. “Laboratory Procedure Manual” (2016).

23. American Diabetes Association, 2. "Classification and Diagnosis of Diabetes". Diabetes Care 38 (2015): S8.

24. Centers for Disease Control and Prevention National Center for Health Statistics. Physical Activity: 2015-2016 Data Documentation, Codebook, and Frequencies (2017).

25. Centers for Disease Control and Prevention and National Center for Health Statistics. National Health and Nutrition Examination Survey: Analytic Guidelines, 2011-2016 (2018).

26. Statistical Analysis System (SAS) software. SAS Institute: Cary, NC, USA (2017).
27. SUDAAN. "RTI International”. Research Triangle Park, NC, USA (2018).

28. Sheard NF., et al. "Dietary carbohydrate (amount and type) in the prevention and management of diabetes: a statement by the american diabetes association". Diabetes Care 27.9 (2004): 2266-2271.

29. Evert AB., et al. "Nutrition therapy for adults with diabetes or prediabetes: A consensus report”. DIabetes Caer 42.5 (2019): 731-754.

30. American Diabetes Association. "Lifestyle Management: Standards of Medical Care in Diabetes-2018". Diabetes Care 41 (2018): S38-S50.

31. Sainsbury E., et al. "Effect of dietary carbohydrate restriction on glycemic control in adults with diabetes: A systematic review and meta-analysis". Diabetes Research and Clinical Practice 139 (2018): 239-252.

32. Hu FB., et al. "Diet, lifestyle, and the risk of type 2 diabetes mellitus in women". The New England Journal of Medicine 345.11 (2001): 790-797.

33. de Koning L., et al. "Diet-quality scores and the risk of type 2 diabetes in men". Diabetes Care 34.5 (2011): 1150-1156.

34. Althubaiti A. "Information bias in health research: definition, pitfalls, and adjustment methods". Journal of Multidisciplinary Healthcare 9 (2016): 211-217.

35. Ye F., et al. "Comparison of patient report and medical records of comorbidities: Results from a population-based cohort of patients with prostate cancer". JAMA Oncology 3.8 (2017): 1035-1042.

36. Pit SW., et al. "Accuracy of telephone self-report of drug use in older people and agreement with pharmaceutical claims data". Drugs Aging 25.1 (2008): 71-80.

\section{Volume 5 Issue 8 August 2021 (C) All rights are reserved by Elta Charles and Eunkyung Lee.}

